\title{
EVERY STATE ON INTERVAL EFFECT ALGEBRA IS INTEGRAL
}

\author{
ANATOLIJ DVUREČENSKIJ \\ 1 Mathematical Institute, Slovak Academy of Sciences \\ Štefánikova 49, SK-814 73 Bratislava, Slovakia \\ E-mail: dvurecen@mat.savba.sk,
}

\begin{abstract}
We show that every state on an interval effect algebra is an integral through some regular Borel probability measure defined on the Borel $\sigma$-algebra of a compact Hausdorff simplex. This is true for every effect algebra satisfying (RDP) or for every MV-algebra. In addition, we show that each state on an effect subalgebra of an interval effect algebra $E$ can be extended to a state on $E$. Our method represents also every state on the set of effect operators of a Hilbert space as an integral.
\end{abstract}

\section{INTRODUCTION}

Effect algebras were defined by Foulis and Bennett in FoBe in order to axiomatize some measurements in quantum mechanics that are modeled by POV-measures as partial algebras with a partially defined addition, + , an analogue of join of two orthogonal elements. Effect algebras generalize Boolean algebras, orthomodular lattices and posets, orthoalgebras as well as MV-algebras. Because Hilbert space quantum mechanics "lives" in a Hilbert space, $H$, the system of all Hermitian operators, $\mathcal{B}(H)$, the system of all effect operators, $\mathcal{E}(H)$, i.e. the set of all Hermitian operators between the zero operator and the identity, as well as the system of all orthogonal projectors, $\mathcal{P}(H)$, are crucial algebraic structures: $\mathcal{B}(H)$ is a po-group that is an antilattice, [LuZa, Thm 58.4], i.e. only comparable elements have join, $\mathcal{E}(H)$ is an effect algebra, and $\mathcal{P}(H)$ is a complete orthomodular lattice. They are fundamental examples of quantum structures; more on quantum structures see $\mathrm{DvPu}$.

We note that effect algebras are equivalent to D-posets defined by Kôpka and Chovanec $\mathrm{KoCh}$, where a primary notion is difference of comparable events.

An important subfamily of effect algebras are MV-algebras introduced by Chang Cha that model many-valued Łukasiewicz logic. The role of MV-algebras for effect algebras is analogous as that of Boolean algebras for orthomodular posets: any orthomodular poset or a lattice effect algebra can be covered by a system of

\footnotetext{
${ }^{1}$ Keywords: Effect algebra; Riesz Decomposition Property; state; unital po-group; simplex; Bauer simplex

AMS classification: 81P15, 03G12, 03B50

The author thanks for the support by Center of Excellence SAS - Quantum Technologies -, ERDF OP R\&D Projects CE QUTE ITMS 26240120009 and meta-QUTE ITMS 26240120022, the grant VEGA No. 2/0032/09 SAV and by the Slovak Research and Development Agency under the contract No. APVV-0071-06, Bratislava.
} 
mutually compatible substructures that in the first case are Boolean subalgebras whilst in the second case are MV-subalgebras.

Many useful effect algebras are intervals in some unital po-group groups. Such examples are effect algebras with the Riesz Decomposition Property (RDP for short), or unigroups or MV-algebras.

A state is an analogue of a probability measure for quantum structures. Roughly speaking, it is a positive functional $s$ on an effect algebra $E$ that preserves + and $s(1)=1$ where 1 is the greatest element in $E$. In general, an effect algebra may have no state, but effect algebra with (RDP) or an interval one has at least one state.

The system of all states is always a convex compact Hausdorff space whose the set of extremal states is not necessarily compact. Anyway, using some analysis of compact convex sets it is possible to show that if $E$ is an interval effect algebra or even if it satisfies (RDP) or an MV-algebra, then a state is an integral through a regular Borel probability measure defined on the Borel $\sigma$-algebra over a compact Hausdorff simplex. This result generalizes the analogous results by [Kro, Kro1] and Panti [Pan] proved for states on MV-algebras. We show also conditions when, for every state $s$ on $E$, there is a unique regular Borel probability measure.

In addition, we generalize Horn-Tarski Theorem that says that every state on a Boolean subalgebra of a Boolean algebra $B$ can be extended to a state on $B$. We show that every state on an effect subalgebra of an interval effect algebra $E$ can be extended to a state on $E$.

The article is organized as follows. Section 2 presents elements of effect algebras, and states on effect algebras are recalled in Section 3. Section 4 is devoted to affine continuous functions on a convex compact Hausdorff topological space and Section 5 shows importance of Choquet and Bauer simplices for our study. Finally, the main results are formulated and proved in Section 6. In Conclusion, we summarize our results.

\section{Elements of Effect Algebras}

An effect algebra is by $\mathrm{FoBe}$ a partial algebra $E=(E ;+, 0,1)$ with a partially defined operation + and two constant elements 0 and 1 such that, for all $a, b, c \in E$,

(i) $a+b$ is defined in $E$ if and only if $b+a$ is defined, and in such a case $a+b=b+a$

(ii) $a+b$ and $(a+b)+c$ are defined if and only if $b+c$ and $a+(b+c)$ are defined, and in such a case $(a+b)+c=a+(b+c)$;

(iii) for any $a \in E$, there exists a unique element $a^{\prime} \in E$ such that $a+a^{\prime}=1$;

(iv) if $a+1$ is defined in $E$, then $a=0$.

We recommend $\mathrm{DvPu}$ for more details on effect algebras.

If we define $a \leq b$ if and only if there exists an element $c \in E$ such that $a+c=b$, then $\leq$ is a partial order on $E$, and we denote $c:=b-a$. Then $a^{\prime}=1-a$ for any $a \in E$.

We will assume that $E$ is not degenerate, i.e. $0 \neq 1$.

Let $E$ and $F$ be two effect algebras. A mapping $h: E \rightarrow F$ is said to be a homomorphism if (i) $h(a+b)=h(a)+h(b)$ whenever $a+b$ is defined in $E$, and (ii) $h(1)=1$. A bijective homomorphism $h$ such that $h^{-1}$ is a homomorphism is said to be an isomorphism of $E$ and $F$. 
Let $\mathcal{E}$ be a system of $[0,1]$-valued functions on $\Omega \neq \emptyset$ such that (i) $1 \in \mathcal{E}$, (ii) $f \in \mathcal{E}$ implies $1-f \in \mathcal{E}$, and (iii) if $f, g \in \mathcal{E}$ and $f(\omega) \leq 1-g(\omega)$ for any $\omega \in \Omega$, then $f+g \in \mathcal{E}$. Then $\mathcal{E}$ is an effect algebra of [0,1]-valued functions, called an effect-clan, that is not necessarily a Boolean algebra nor an MV-algebra.

We say that an effect algebra $E$ satisfies the Riesz Decomposition Property, (RDP) for short, if $x \leq y_{1}+y_{2}$ implies that there exist two elements $x_{1}, x_{2} \in E$ with $x_{1} \leq y_{1}$ and $x_{2} \leq y_{2}$ such that $x=x_{1}+x_{2}$.

We recall that $E$ has (RDP) if and only if, [DvPu, Lem. 1.7.5], $x_{1}+x_{2}=y_{1}+y_{2}$ implies there exist four elements $c_{11}, c_{12}, c_{21}, c_{22} \in E$ such that $x_{1}=c_{11}+c_{12}$, $x_{2}=c_{21}+c_{22}, y_{1}=c_{11}+c_{21}$, and $y_{2}=c_{12}+c_{22}$.

A partially ordered Abelian group $(G ;+, 0)$ (po-group in short) is said to satisfy interpolation provided given $x_{1}, x_{2}, y_{1}, y_{2}$ in $G$ such that $x_{1}, x_{2} \leq y_{1}, y_{2}$ there exists $z$ in $G$ such that $x_{1}, x_{2} \leq z \leq y_{1}, y_{2}$. We say also that $G$ is an interpolation group.

A po-group $(G, u)$ with strong unit $u$ (= order unit, i.e. given $g \in G$, there is an integer $n \geq 1$ such that $g \leq n$ ) is said to be a unital po-group. If the set

$$
\Gamma(G, u):=\{g \in G: 0 \leq g \leq u\}
$$

is endowed with the restriction of the group addition + , then $(\Gamma(G, u) ;+, 0, u)$ is an effect algebra.

Ravindran $\mathrm{Rav}$ ( $\mathrm{DvPu}, \mathrm{Thm}$ 1.7.17]) showed that every effect algebra with (RDP) is of the form (2.1) for some interpolation unital po-group $(G, u)$ :

Theorem 2.1. Let $E$ be an effect algebra with (RDP). Then there exists a unique (up to isomorphism of Abelian po-groups with strong unit) interpolation group $(G, u)$ with strong unit such that $\Gamma(G, u)$ is isomorphic with $E$.

Moreover, the category of effect algebras with (RDP) is categorically equivalent with the category of unital po-groups with interpolation; the categorical equivalence is given by the functor: $\Gamma:(G, u) \mapsto \Gamma(G, u)$.

An effect algebra of the form $\Gamma(G, u)$ for some element $u \geq 0$ or if it is isomorphic with $\Gamma(G, u)$ is called also an interval effect algebra. For example, the system, $\mathcal{E}(H)$, of all Hermitian operators, $A$, of a Hilbert space $H$ (real, complex or quaternionic) such that $O \leq A \leq I$, where the ordering of Hermitian operators is defined by the property: $A \leq B$ iff $(A \phi, \phi) \leq(B \phi, \phi)$ for all $\phi \in H$, is an interval effect algebra such that $\mathcal{E}(H)=\Gamma(\mathcal{B}(H), I)$. We note that $\mathcal{E}(H)$ is without (RDP).

Let $u$ be a positive element of an Abelian po-group $G$. The element $u$ is said to be generative if every element $g \in G^{+}$is a group sum of finitely many elements of $\Gamma(G, u)$, and $G=G^{+}-G^{+}$. Such an element is a strong unit [DvPu, Lem 1.4.6] for $G$ and the couple $(G, u)$ is said to be a po-group with generative strong unit. For example, if $u$ is a strong unit of an interpolation po-group $G$, then $u$ is generative. The same is true for $I$ and $\Gamma(\mathcal{B}(H), I)$.

Let $E$ be an effect algebra and $H$ be an Abelian (po-) group. A mapping $p$ : $G \rightarrow H$ that preserves + is called an $H$-valued measure on $E$.

Remark 2.2. If $E$ is an interval effect algebra, then there is a po-group $G$ with a generative strong unit $u$ such that $E \cong \Gamma(G, u)$ and every $H$-valued measure $p: \Gamma(G, u) \rightarrow H$ can be extended to a group-homomorphism $\phi$ from $G$ into $H$. If $H$ is a po-group, then $\phi$ is a po-group-homomorphism. Then $\phi$ is unique and $(G, u)$ is also unique up to isomorphism of unital (po-) groups, see [ $\mathrm{DvPu}$, Cor 1.4.21]; the element $u$ is said to be a universal strong unit for $\Gamma(G, u)$ and the couple $(G, u)$ is 
said to be a unigroup. In particular, the identity operator $I$ is a universal strong unit for $\Gamma(\mathcal{B}(H), I)$, $\mathrm{DvPu}$, Cor 1.4.25], similarly for $\Gamma(A, I)$, where $A$ is a von Neumann algebra on $H$.

Also in this case, the category of interval effect algebras is categorically equivalent with the category of unigroups $(G, u)$ with the functor $\Gamma:(G, u) \mapsto \Gamma(G, u)$.

We notify that also a non-commutative version of effect algebras, called pseudo effect algebras, was recently introduced in DvVe1, DvVe2; in some important cases they are also intervals but in unital po-groups that are not necessarily Abelian.

A very important family of effect algebras is the family of MV-algebras, which were introduced by Chang Cha:

An $M V$-algebra is an algebra $\left(M ; \oplus,{ }^{*}, 0\right)$ of signature $\langle 2,1,0\rangle$, where $(M ; \oplus, 0)$ is a commutative monoid with neutral element 0 , and for all $x, y \in M$

(i) $\left(x^{*}\right)^{*}=x$,

(ii) $x \oplus 1=1$, where $1=0^{*}$,

(iii) $x \oplus\left(x \oplus y^{*}\right)^{*}=y \oplus\left(y \oplus x^{*}\right)^{*}$.

We define also an additional total operation $\odot$ on $M$ via $x \odot y:=\left(x^{*} \oplus y^{*}\right)^{*}$. For more on MV-algebras, see the monograph [CDM].

If we define a partial operation + on an MV-algebra $M$ in such a way that $a+b$ is defined in $M$ if and only if $a \leq b^{*}$ (or equivalently $a \odot b=0$ ) and we set $a+b:=a \oplus b$, then $(M ;+, 0,1)$ is a lattice ordered effect algebra with (RDP), where $a^{\prime}:=a^{*}$. According to Mun, every MV-algebra is isomorphic with $\left(\Gamma(G, u) ; \oplus,{ }^{*}, 0\right)$, where $(G, u)$ is an $\ell$-group (= lattice ordered group) with strong unit and

$$
a \oplus b=(a+b) \wedge u, \quad a^{*}=u-a, \quad a, b \in \Gamma(G, u) .
$$

It is worthy recalling that a lattice ordered effect algebra $E$ with (RDP) can be converted into an MV-algebra (in such a way that both original + and the partial addition induced from the MV-structure coincide); we define $x \oplus y:=x+\left(y \wedge(x \wedge y)^{\prime}\right)$ and $x^{*}=x^{\prime}$, see $[\mathrm{DvPu}$, Thm 1.8.12].

\section{States on Effect Algebras}

We present some notions and properties about states on effect algebras. For further reading we recommend $\mathrm{DvPu}, \mathrm{Dvu}$.

A state on an effect algebra $E$ is any mapping $s: E \rightarrow[0,1]$ such that (i) $s(1)=1$, and (ii) $s(a+b)=s(a)+s(b)$ whenever $a+b$ is defined in $E$. We denote by $\mathcal{S}(E)$ the set of all states on $E$. It can happen that $\mathcal{S}(E)$ is empty, see e.g. DvPu, Exam. 4.2.4]. In important cases, for example, when $E$ satisfies (RDP) or, more generally, if $E$ is an interval effect, $\mathcal{S}(E)$ is nonempty, see below. $\mathcal{S}(E)$ is always a convex set, i.e. if $s_{1}, s_{2}$ are states on an effect algebra $E$ and $\lambda \in[0,1]$, then $s=\lambda s_{1}+(1-\lambda) s_{2}$ is a also a state on $E$. A state $s$ is said to be extremal if $s=\lambda s_{1}+(1-\lambda) s_{2}$ for $\lambda \in(0,1)$ implies $s=s_{1}=s_{2}$. By $\partial_{e} \mathcal{S}(E)$ we denote the set of all extremal states of $\mathcal{S}(E)$. We say that a net of states, $\left\{s_{\alpha}\right\}$, on $E$ weakly converges to a state $s$ on $E$ if $s_{\alpha}(a) \rightarrow s(a)$ for any $a \in E$. In this topology, $\mathcal{S}(E)$ is a compact Hausdorff topological space and every state on $E$ belongs to the weak closure of the convex hull of the extremal states, as the Krein-Mil'man Theorem states, see e.g. [Goo, Thm 5.17].

Let $(G, u)$ be an Abelian po-group with strong unit. By a state on $(G, u)$ we mean any mapping $s: G \rightarrow \mathbb{R}$ such that (i) $s(g+h)=s(g)+s(h)$ for all $g, h \in G$, (ii) $s\left(G^{+}\right) \subseteq \mathbb{R}^{+}$, and (iii) $s(u)=1$. In other words, a state on $(G, u)$ is any 
po-group homomorphism from $(G, u)$ into the po-group $(\mathbb{R}, 1)$ that preserves fixed strong units. We denote by $\mathcal{S}(G, u)$ the set of all states and by $\partial_{e} \mathcal{S}(G, u)$ the set of all extremal states, respectively, on $(G, u)$. According to [Goo, Cor. 4.4], $\mathcal{S}(G, u) \neq \emptyset$ whenever $u>0$. In a similar way as for effect algebras, we define the weak convergence of states on $(G, u)$, and analogously, $\mathcal{S}(G, u)$ is a compact Hausdorff topological space and every state on $(G, u)$ is a weak limit of a net of convex combinations from $\partial_{e} \mathcal{S}(G, u)$.

If $E=\Gamma(G, u)$, where $(G, u)$ is an interpolation po-group or a unigroup, then due to the categorical equivalence, every state on $E$ can be extended to a unique state on $(G, u)$, and conversely, the restriction of any state on $(G, u)$ to $E$ gives a state on $E$. Therefore, if $E$ satisfies (RDP) or more generally if $E$ is an interval effect algebra, $\mathcal{S}(E) \neq \emptyset$. In addition, extremal states on $E$ are the restrictions of extremal states on $(G, u)$, the space $\mathcal{S}(E)$ is affinely homeomorphic with $\mathcal{S}(G, u)$, and the space $\partial_{e} \mathcal{S}(E)$ is homeomorphic with $\partial_{e} \mathcal{S}(G, u)$. We recall that a mapping from one convex set onto another convex set is affine if it preserves convex combinations.

We recall that if $E$ is an MV-algebra, then a state $s$ on $E$ is extremal if and only if $s(x \wedge y)=\min \{s(x), s(y)\}, x, y \in E$, therefore $\partial_{e} \mathcal{S}(E)$ is compact (see, e.g. DvPu, Prop. 6.1.19], [Goo, Thm 12.18]).

On the other hand, if $E$ is an effect algebra with (RDP), then $\partial_{e} \mathcal{S}(E)$ is not necessarily compact, see for example [Goo, Exam. 6.10].

We say that a system of states, $\mathcal{S}$, on an effect algebra $E$ is order determining if $s(a) \leq s(b)$ for any $s \in \mathcal{S}$ yields $a \leq b$.

Let $H$ be a separable Hilbert space (real, complex or quaternionic) with an inner product $(\cdot, \cdot)$, and $\mathcal{L}(H)$ be the system of all closed subspaces of $H$. Then $\mathcal{L}(H)$ is a complete orthomodular lattice Dvu. Given a unit vector $\phi \in H$, let

$$
p_{\phi}(M):=\left(P_{M} \phi, \phi\right), \quad M \in \mathcal{L}(H),
$$

where $P_{M}$ is the orthogonal projector of $H$ onto $M$. Then $p_{\phi}$ is a $\sigma$-additive state on $\mathcal{L}(H)$, called a pure state. The system of all pure states is order determining. If $T$ is a positive Hermitian operator of finite trace (i.e. $\sum_{i}\left(T \phi_{i}, \phi_{i}\right)<\infty$ for any orthonormal basis $\left\{\phi_{i}\right\}$ of $H$, and we define $\left.\operatorname{tr}(T):=\sum_{i}\left(T \phi_{i}, \phi_{i}\right)\right)$, then

$$
s_{T}(M):=\operatorname{tr}\left(T P_{M}\right), \quad M \in \mathcal{L}(H),
$$

is a $\sigma$-additive state, and according to famous Gleason's Theorem, Gle, Dvu, Thm 3.2.24], if $\operatorname{dim} H \geq 3$, for every $\sigma$-additive state $s$ on $\mathcal{L}(H)$, there is a unique positive Hermitian operator $T$ of finite trace such that $s=s_{T}$.

If now $s$ is a finitely additive state on $\mathcal{L}(H)$, then due to the Aarnes Theorem, Dvu, Thm 3.2.28], $s$ can be uniquely decomposed to the form

$$
s=\lambda s_{1}+(1-\lambda) s_{2},
$$

where $s_{1}$ is a $\sigma$-additive state and $s_{2}$ is a finitely additive state vanishing on each finite-dimensional subspace of $H$.

Moreover, a pure state $p_{\phi}$ is an extreme point of the set of $\sigma$-additive states, as well as it is an extremal state of $\mathcal{L}(H)$. Other extremal states of $\mathcal{L}(H)$ vanish on each finite-dimensional subspace of $H$. Since every state on $\mathcal{L}(H)$ can be extended into a unique state on $\mathcal{B}(H)$, see e.g. [Dvu, Thm 3.3.1], the state spaces $\mathcal{S}(\mathcal{L}(H)), \mathcal{S}(\mathcal{E}(H))$ and $\mathcal{S}(\mathcal{B}(H))$ are mutually affinely homeomorphic whenever $3 \leq \operatorname{dim} H \leq \aleph_{0}$.

It is worthy to recall that $\mathcal{L}(H)$ is isomorphic to the set $\mathcal{P}(H)$ of all orthogonal projectors of $H$. 
We say that a po-group $G$ is Archimedean if for $x, y \in G$ such that $n x \leq y$ for all positive integers $n \geq 1$, then $x \leq 0$. It is possible to show that a unital po-group $(G, u)$ is Archimedean iff $G^{+}=\{g \in G: s(g) \geq 0$ for all $s \in \mathcal{S}(G, u)\}$, Goo, Thm 4.14]. We have the following characterization of interval effect algebras coming from Archimedean po-groups.

Proposition 3.1. Let $E=\Gamma(G, u)$, where is $(G, u)$ is a unigroup. The following statements are equivalent:

(i) $\mathcal{S}(E)$ is order determining.

(ii) $E$ is isomorphic to some effect-clan.

(iii) $G$ is Archimedean.

Proof. (i) $\Rightarrow$ (ii). Given $a \in E$, let $\hat{a}$ be a function from $\mathcal{S}(E)$ into the real interval $[0,1]$ such that $\hat{a}(s):=s(a)$ for any $s \in \mathcal{S}(E)$, and let $\hat{E}=\{\hat{a}: a \in E\}$. We endow $\hat{E}$ with pointwise addition, so that $\hat{E}$ is an effect-clan. Since $\mathcal{S}(E)$ is order determining, the mapping $a \mapsto \hat{a}$ is an isomorphism and $\hat{E}$ is Archimedean.

(ii) $\Rightarrow$ (iii). Let $\hat{E}$ be any effect-clan isomorphic with $E$, and let $a \mapsto \hat{a}$ be such an isomorphism. Let $G(\hat{E})$ be the po-group generated by $\hat{E}$. Then $G(\hat{E})$ consists of all functions of the form $\hat{a}_{1}+\cdots+\hat{a}_{n}-\hat{b}_{1}-\cdots-\hat{b}_{m}$, and $\hat{1}$ is its strong unit. Due to the categorical equivalence, $(G, u)$ and $(G(\hat{E}), \hat{1})$ are isomorphic. But $(G(\hat{E}), \hat{1})$ is Archimedean, so is $(G, u)$.

(iii) $\Rightarrow$ (i). Due to [Goo, Thm 4.14], $G^{+}=\{g \in G: s(g) \geq 0$ for all $s \in \mathcal{S}(G, u)\}$, which means that $\mathcal{S}(G, u)$ is order determining. Hence, the restrictions of all states on $(G, u)$ onto $E=\Gamma(G, u)$ entail $\mathcal{S}(E)$ is order determining.

\section{Affine Continuous Functions}

We present some important notions about affine continuous functions that are important for theory of effect algebras. A good source for this topic are monographs Goo, Alf.

Let $K$ be a convex subset of a real vector space $V$. A point $x \in K$ is said to be extreme if from $x=\lambda x_{1}+(1-\lambda) x_{2}$, where $x_{1}, x_{2} \in K$ and $0<\lambda<1$ we have $x=x_{1}=x_{2}$. By $\partial_{e} K$ we denote the set of extreme points of $K$, and if $K$ is a compact convex subset of a locally convex Hausdorff topological space, then due to the Krein-Mil'man Theorem [Goo, Thm 5.17], $K$ equals to the closure of the convex hull of $\partial_{e} K$, i.e. $K=\left(\operatorname{con}\left(\partial_{e} K\right)\right)^{-}$, where ${ }^{-}$and con denote the closure and the convex hull, respectively.

A mapping $f: K \rightarrow \mathbb{R}$ is said to be affine if, for all $x, y \in K$ and any $\lambda \in[0,1]$, we have $f(\lambda x+(1-\lambda) y)=\lambda f(x)+(1-\lambda) f(y)$.

Given a compact convex set $K$ in a topological vector space, we denote by $\operatorname{Aff}(K)$ the set of all affine continuous functions on $K$. Then $\operatorname{Aff}(K)$ is a po-subgroup of the po-group $\mathrm{C}(K)$ of all continuous real-valued functions on $K$ (we recall that, for $f, g \in \mathrm{C}(K), f \leq g$ iff $f(x) \leq g(x)$ for any $x \in K)$, hence it is an Archimedean unital po-group with the strong unit 1 . In addition, $\mathrm{C}(K)$ is an $\ell$-group (= lattice ordered group).

For example, if $E$ is an effect algebra such that its state space is nonempty, then for any $a \in E$, the function $\hat{a}(s)=s(a), s \in \mathcal{S}(E)$, is a continuous affine function belonging to $\operatorname{Aff}(\mathcal{S}(E))$. 
In contrast to $\mathrm{C}(K)$, the space $\operatorname{Aff}(K)$ is not necessarily a lattice. Indeed, according to Goo, Thm 11.21], it is possible to show that if $(G, u)$ is a unital interpolation group, then $\operatorname{Aff}(\mathcal{S}(G, u))$ is a lattice if and only if $\partial_{e} \mathcal{S}(G, u)$ is compact.

Let now $K$ be a compact convex subset of a locally convex Hausdorff space, and let $S=\mathcal{S}(\operatorname{Aff}(K), 1)$. Then the evaluation mapping $\psi: K \rightarrow S$ defined by $\psi(x)(f)=f(x)$ for all $f \in \operatorname{Aff}(K)(x \in K)$ is an affine homeomorphism of $K$ onto $S$, see Goo, Thm 7.1].

Let $\mathcal{B}_{0}(K)$ be the Baire $\sigma$-algebra, i.e. the $\sigma$-algebra generated by compact $G_{\delta^{-}}$ sets (a $G_{\delta}$-set is a countable intersection of open sets) of a compact set $K$, and the elements of $\mathcal{B}_{0}(K)$ are said to be Baire sets, and a $\sigma$-additive (signed) measure of $\mathcal{B}_{0}(K)$ is a Baire measure.

Similarly, $\mathcal{B}(K)$ is the Borel $\sigma$-algebra of $K$ generated by all open subsets of $K$ and any element of $\mathcal{B}(K)$ is said to be a Borel set, and any $\sigma$-additive (signed) measure on it is said to be a Borel measure. It is well-known that each Baire measure can be extended to a unique regular Borel measure.

Let $\mathcal{M}(K)$ denote the set of all finite signed regular Borel measures on $\mathcal{B}(K)$ and let $\mathcal{M}_{1}^{+}(K)$ denote the set of all probability measures, that is, all positive regular Borel measures $\mu \in \mathcal{M}(K)$ such that $\mu(K)=1$. We recall that a Borel measure $\mu$ is called regular if

$$
\inf \{\mu(O): Y \subseteq O, O \text { open }\}=\mu(Y)=\sup \{\mu(C): C \subseteq Y, C \text { closed }\}
$$

for any $Y \in \mathcal{B}(K)$.

Due to the Riesz Representation Theorem, DuSc], we can identify the set $\mathcal{M}(K)$ with the dual of the space $\mathrm{C}(K)$ by setting

$$
\mu(f)=\int_{K} f \mathrm{~d} \mu
$$

for all $\mu \in \mathcal{M}(K)$ and all $f \in \mathrm{C}(K)$.

We endow $\mathcal{M}(K)$ with the weak* topology, i.e., a net $\mu_{\alpha}$ converges to an element $\mu \in \mathcal{M}(K)$ iff $\mu_{\alpha}(f) \rightarrow \mu(f)$ for all $f \in \mathrm{C}(K)$.

Let $x$ be a fixed point in $K$. Then there is a unique Borel regular measure $\mu=\mu_{x}$ (depending on $x$ ) such that

$$
f(x)=\int_{K} f \mathrm{~d} \mu, f \in \mathrm{C}(K) .
$$

Let $\delta_{x}$ be the Dirac measure concentrated at the point $x \in K$, i.e., $\delta_{x}(Y)=1$ iff $x \in Y$, otherwise $\delta_{x}(Y)=0$; then every Dirac measure is a regular Borel probability measure. In formula (4.2) we have then $\mu_{x}=\delta_{x}$. Moreover, [Goo, Prop 5.24], the mapping

$$
\epsilon: x \mapsto \delta_{x}
$$

gives a homeomorphism of $K$ onto $\partial_{e} \mathcal{M}_{1}^{+}(K)$.

Suppose that $K$ is a nonempty compact subset of a locally convex space $V$, and let $\mu \in \mathcal{M}_{1}^{+}(K)$. A point $x$ in $V$ is said to be represented by $\mu$ (or $x$ is the barycenter of $\mu$ ) if

$$
f(x)=\int_{K} f \mathrm{~d} \mu, \quad f \in V^{*},
$$

where $V^{*}$ stands for the set of continuous linear functionals $f$ in $V$. 
From the Krein-Mil'man Theorem, it is possible to derive [Phe, p. 6], that every point $x$ of a compact convex set $K$ of a locally convex space $V$ is the barycenter of a probability measure $\mu \in \mathcal{M}_{1}^{+}(K)$ such that $\mu\left(\left(\partial_{e} K\right)^{-}\right)=1$.

We note that according to a theorem by Bauer [Phe, Prop 1.4], if $K$ is a convex compact subset of a locally convex space $V$, then a point $x$ is an extreme point of $K$ iff the Dirac measure $\delta_{x}$ is a unique probability measure from $\mathcal{M}_{1}^{+}(K)$ whose barycenter is $x$.

\section{Choquet Simplices}

We show that, for theory of effect algebras, simplices are important, for more about simplices see in Goo, Alf, Phe. In what follows, for the reader convenience, we present some necessary notions and results on Choquet and Bauer simplices.

We recall that a convex cone in a real linear space $V$ is any subset $C$ of $V$ such that (i) $0 \in C$, (ii) if $x_{1}, x_{2} \in C$, then $\alpha_{1} x_{1}+\alpha_{2} x_{2} \in C$ for any $\alpha_{1}, \alpha_{2} \in \mathbb{R}^{+}$. A strict cone is any convex cone $C$ such that $C \cap-C=\{0\}$, where $-C=\{-x: x \in C\}$. A base for a convex cone $C$ is any convex subset $K$ of $C$ such that every non-zero element $y \in C$ may be uniquely expressed in the form $y=\alpha x$ for some $\alpha \in \mathbb{R}^{+}$and some $x \in K$.

We recall that in view of [Goo, Prop 10.2], if $K$ is a non-void convex subset of $V$, and if we set

$$
C=\left\{\alpha x: \alpha \in \mathbb{R}^{+}, x \in K\right\},
$$

then $C$ is a convex cone in $V$, and $K$ is base of $C$ iff there is a linear functional $f$ on $V$ such that $f(K)=1$ iff $K$ is contained in a hyperplane in $V$ which misses the origin.

Any strict cone $C$ of $V$ defines a partial order $\leq_{C}$ via $x \leq_{C} y$ iff $y-x \in C$. It is clear that $C=\left\{x \in V: 0 \leq_{C} x\right\}$. A lattice cone is any strict convex cone $C$ in $V$ such that $C$ is a lattice under $\leq_{C}$.

A simplex in a linear space $V$ is any convex subset $K$ of $V$ that is affinely isomorphic to a base for a lattice cone in some real linear space. A simplex $K$ in a locally convex Hausdorff space is said to be (i) Choquet if $K$ is compact, and (ii) Bauer if $K$ and $\partial_{e} K$ are compact.

For example, for the important quantum mechanical example, if $H$ is a separable complex Hilbert space, $\mathcal{S}(\mathcal{E}(H))$ is not a simplex due to [AlSc, Thm 4.4], where it is shown that the state space of $\mathcal{S}(\mathcal{E}(H))$ for the two-dimensional $H$ is isomorphic to the unit three-dimensional real ball, consequently $\mathcal{S}(\mathcal{E}(H)$ ) is not a simplex whenever $\operatorname{dim} H>1$. Of course, $\mathcal{E}(H)$ does not satisfy (RDP):

Theorem 5.1. If an effect algebra $E$ satisfies (RDP), then $\mathcal{S}(E)$ is a Choquet simplex.

Proof. In view of Theorem 2.1 there is a unital po-group $(G, u)$ with interpolation such that $E=\Gamma(G, u)$. Since $\mathcal{S}(E)$ is affinely homeomorphic with $\mathcal{S}(G, u)$, we have that $\mathcal{S}(E)$ is a Choquet simplex iff so is $\mathcal{S}(G, u)$. By [Goo, Thm 10.17], $\mathcal{S}(G, u)$ is a Choquet simplex. We recall that $\mathcal{S}(G, u)$ is a base for the positive cone of the Dedekind complete lattice-ordered real vector space of all relatively bounded homomorphisms from $G$ to $\mathbb{R}$.

Example 5.2. If $E$ is an interval effect algebra that does not satisfies (RDP), then $\mathcal{S}(E)$ is not necessarily a Choquet simplex. 
Indeed, let $G=\left\{\left(x_{1}, x_{2}, x_{3}, x_{4}\right) \in \mathbb{Z}^{4}: x_{1}+x_{2}=x_{3}+x_{4}\right\}, u=(1,1,1,1)$, and $E=\Gamma(G, u)$. If $s_{i}\left(x_{1}, x_{2}, x_{3}, x_{4}\right)=x_{i}$, then $\partial_{e} \mathcal{S}(E)=\left\{s_{1}, s_{2}, s_{3}, s_{4}\right\}$ and $\mathcal{S}(E)$ is not a simplex because $\left(s_{1}+s_{2}\right) / 2=\left(s_{3}+s_{4}\right) / 2$, see also [Goo, Cor 10.8].

Another example is $\mathcal{S}(\mathcal{E}(H))$ for the effect algebra $\mathcal{E}(H)$.

The importance of Choquet and Bauer simplices follows from the fact that if $K$ is a convex compact subset of a locally convex Hausdorff space, then $K$ is a Choquet simplex iff $(\operatorname{Aff}(K), 1)$ is an interpolation po-group, Goo, Thm 11.4], and $K$ is a Bauer simplex iff $(\operatorname{Aff}(K), 1)$ is an $\ell$-group, Goo, Thm 11.21]. Consequently, there is no MV-algebra whose state space is affinely isomorphic to the closed square or the closed unit circle.

For two measures $\mu$ and $\lambda$ we write

$$
\mu \sim \lambda \quad \text { iff } \quad \mu(f)=\lambda(f), f \in \operatorname{Aff}(K) .
$$

If $\mu$ and $\lambda$ are nonnegative regular Borel measures on $K$, write

$$
\mu \prec \lambda \quad \text { iff } \quad \mu(f) \leq \lambda(f), f \in \operatorname{Con}(K),
$$

where $\operatorname{Con}(K)$ is the set of all continuous convex functions $f$ on $K$ (that is $f\left(\alpha x_{1}+\right.$ $\left.(1-\alpha) x_{2}\right) \leq \alpha f\left(x_{1}\right)+(1-\alpha) f\left(x_{2}\right)$ for $x_{1}, x_{2} \in K$ and $\left.\alpha \in[0,1]\right)$. Then $\prec$ is a partial order on the cone of nonnegative measures. The fact $\lambda \prec \mu$ and $\mu \prec \lambda$ implies $\lambda=\mu$ follows from the fact that $\operatorname{Con}(K)-\operatorname{Con}(K)$ is dense in $\mathrm{C}(K)$.

Moreover, for any probability measure (= regular Borel probability measure) $\lambda$ there is a maximal probability measure $\mu$ such that $\mu \succ \lambda$, [Phe, Lem 4.1], and every point $x \in K$ can be represented by a maximal probability measure.

From the Bishop-de Leeuw Theorem, [Alf, Cor I.4.12], see also [Phe, p. 24], it follows that if $\mu$ is a maximal probability measure on $\mathcal{B}(K)$, then for any Baire set $B$ disjoint with $\partial_{e} K, \mu(B)=0$. In general, the statement is not true if we suppose that $B$ is Borel instead Baire.

An important characterization of Choquet simplices is given by Choquet-Meyer, Phe, Thm p. 66], saying that a compact convex subset $K$ of a locally convex space is a Choquet simplex iff, for every point $x \in K$, there is a unique maximal probability measure $\mu_{x}$ such that $\mu_{x} \sim \delta_{x}$. That is,

$$
f(x)=\int_{K} f \mathrm{~d} \mu_{x}, \quad f \in \operatorname{Aff}(K) .
$$

And the characterization by Bauer, [Alf, Thm II.4.1], says that a Choquet simplex $K$ is Bauer iff for any point $x \in K$, there is a unique Baire probability (equivalently, regular Borel) measure $\mu_{x}$ such that $\mu_{x}\left(\overline{\partial_{e} K}\right)=1$ and (5.1) holds.

\section{Effect Algebras with the Bauer State Property}

We present the main results of the paper, namely we show that every state on an interval effect algebra is an integral. We show that in some cases every state corresponds to a unique regular Borel probability measure. In addition, we generalize Horn-Tarski result also for interval effect algebras and its subalgebras.

We say that an effect algebra has the Bauer state property ((BSP), for short) if the state space $\mathcal{S}(E)$ is a Bauer simplex. For example, every lattice ordered effect algebra with (RDP) has (BSP). The next example is a case when also a non-lattice ordered effect algebra with (RDP) has (BSP). The next example is from [Goo, Ex 4.20]. 
Example 6.1. Let $H=\mathbb{Z}$ be a po-group with the discrete ordering and $G=\mathbb{Q} \overrightarrow{\times} H$ be the lexicographic product. Then $G$ has interpolation but $G$ is not a lattice. If we set $u=(1,0)$, then $u$ is a strong unit in $G$, and let $E=\Gamma(G, u)$. If $s$ is a state on $G$, then due to $n(k / n, 0)=u$ we have $s(k / n, 0)=k / n$. On the other hand, $-u \leq n(0, b) \leq u$ so that $-1 \leq n s(0, b) \leq 1$ which proves $s(0, b)=0$. Hence, $s(a, b)=a$ proving that $\mathcal{S}(G, u)$ is a singleton and therefore, $E$ has (BSP). In addition, the direct product $E^{n}$ has also (BSP) for any integer $n \geq 1$.

We note that in our example $\operatorname{Ker}(s)=\{(0, b): b \in H\}$, and then the quotient effect algebra $(G / \operatorname{Ker}(s), u / \operatorname{Ker}(s)) \cong(\mathbb{Q}, 1)$.

Theorem 6.2. Let $E$ be an interval effect algebra and let $s$ be a state on $E$. Let $\psi: E \rightarrow \operatorname{Aff}(\mathcal{S}(E))$ be defined by $\psi(a):=\hat{a}, a \in E$, where $\hat{a}$ is a mapping from $\mathcal{S}(E)$ into $[0,1]$ such that $\hat{a}(s):=s(a), s \in \mathcal{S}(E)$. Then there is a unique state $\tilde{s}$ on the unital po-group $(\operatorname{Aff}(\mathcal{S}(E)), 1)$ such that $\tilde{s}(\hat{a})=s(a)$ for any a $\in$ E. Moreover, this state can be extended to a state on $(C(\mathcal{S}(E), 1))$.

The mapping $s \mapsto \tilde{s}$ defines an affine homeomorphism from the state space $\mathcal{S}(E)$ onto $\mathcal{S}(\Gamma(\operatorname{Aff}(\mathcal{S}(E)), 1))$.

If, in addition, $E$ is has an order system of states, then $\widehat{E}:=\{\hat{a}: a \in E\}$ is an effect-clan that is a sub-effect algebra of $\Gamma(\operatorname{Aff}(\mathcal{S}(E)), 1)$ and every state $s$ on $E$ can be extended to a state $\tilde{s}$ on $\Gamma(C(\mathcal{S}(E)), 1)$ as well as to a state on the unital po-group $(C(\mathcal{S}(E)), 1)$.

Proof. Since $E$ is an interval effect algebra and $E=\Gamma(G, u)$ for some unigroup $(G, u), \mathcal{S}(E)$ is non-void. The mapping $\psi$ can be uniquely extended to a po-group homomorphism $\hat{\psi}: G \rightarrow \operatorname{Aff}(\mathcal{S}(E))$. Let $\hat{s}$ be a state on $(G, u)$ that is a unique extension of a state $s$. Applying now [Goo, Prop 7.20], the statement follows, in particular, we have that there is a unique state $\tilde{s}$ on $(\operatorname{Aff}(\mathcal{S}(E)), 1)$ such that $\tilde{s}(\hat{a})=$ $s(a), a \in E$.

Due to Goo, Cor 4.3], we see that this state can be extended to a state on $(\mathrm{C}(\mathcal{S}(E)), 1)$ as well as to a state on $\Gamma(\mathrm{C}(\mathcal{S}(E)), 1)$.

The affine homeomorphism $s \mapsto \tilde{s}$ follows from [Goo, Thm 7.1].

If $E$ is Archimedean, the result follows from Proposition 3.1 and the first part of the present proof.

Theorem 6.3. Let $E$ be an interval effect algebra such that $\mathcal{S}(E)$ is a simplex and let $s$ be a state on $E$. Then there is a unique maximal regular Borel probability measure $\mu_{s} \sim \delta_{s}$ on $\mathcal{B}(\mathcal{S}(E))$ such that

$$
s(a)=\int_{\mathcal{S}(E)} \hat{a}(x) \mathrm{d} \mu_{s}(x), \quad a \in E .
$$

Proof. Due to our hypothesis, $\mathcal{S}(E)$ is a Choquet simplex. By Theorem 6.2 there is a unique state $\tilde{s}$ on $(\operatorname{Aff}(\mathcal{S}(E)), 1)$ such that $\tilde{s}(\hat{a})=s(a), a \in A$.

Applying the result by Choquet-Meyer, Phe, Thm p. 66], see (5.1), we have

$$
f(s)=\int_{\mathcal{S}(E)} f(x) \mathrm{d} \mu_{s}, \quad f \in \operatorname{Aff}(\mathcal{S}(E)) .
$$

Since $\hat{a} \in \operatorname{Aff}(\mathcal{S}(E))$ for any $a \in E$, we have the statement in question.

We recall that a state $s$ on $E$ is $\sigma$ additive if $a_{n} \nearrow a$, then $\lim _{n} s\left(a_{n}\right)=s(a)$. In the same manner we define a $\sigma$-additive state on $(G, u)$. 
Theorem 6.4. Let $E=\Gamma(G, u)$ be an interval effect algebra where $(G, u)$ is a unigroup, and let $\mathcal{S}(E)$ be a simplex. If $s$ is $\sigma$-additive, then its unique extension, $\hat{s}$, on $(G, u)$ is $\sigma$-additive.

Proof. Let $s$ be a $\sigma$-additive state on $E$ and let $\hat{s}$ be its unique extension onto $(G, u)$. By Theorem 6.3, there is a unique maximal regular Borel probability measure $\mu_{s} \sim \delta_{s}$ such that (6.1) holds.

For any $g \in G$, let $\hat{g}$ denote the function on $\mathcal{S}(E)$ such that $\hat{g}(\hat{s})=\hat{s}(g), s \in \mathcal{S}(E)$, where $\hat{s}$ is a unique extension of $s$ onto $(G, u)$. If $g \in G^{+}$, then $g=g_{1}+\cdots+g_{k}$ for some $g_{1}, \ldots, g_{k} \in E, \hat{g}=\hat{g}_{1}+\cdots+\hat{g}_{k}$, and

$$
\begin{aligned}
\hat{s}(g) & =s\left(g_{1}\right)+\cdots+s\left(g_{k}\right) \\
& =\int_{\mathcal{S}(E)} \hat{g}_{1}(x) \mathrm{d} \mu_{s}(x)+\cdots+\int_{\mathcal{S}(E)} \hat{g}_{k}(x) \mathrm{d} \mu_{s}(x) \\
& =\int_{\mathcal{S}(E)} \hat{g}(x) \mathrm{d} \mu_{s}(x) .
\end{aligned}
$$

The $\sigma$-additivity of $\mu_{s}$ entails due to the Lebesgue Convergence Theorem, [Hal], that if $g_{n} \nearrow g$, then $\lim _{n} \hat{s}\left(g_{n}\right)=\hat{s}(g)$ for any $g \in G^{+}$.

If now $g_{n} \searrow 0$, then $\lim _{n} \hat{s}\left(g_{n}\right)=0$; in fact, there is an integer $k \geq 1$ such that $g_{n} \leq g_{1} \leq k u$ for any $n \geq 1$. This gives $k u-g_{n} \nearrow k u$ and $\lim _{n} \hat{s}\left(k u-g_{n}\right)=\hat{s}(k u)$, so that $\lim _{n} \hat{s}\left(g_{n}\right)=0$.

Finally, let $g \in G$ be arbitrary and let $g_{n} \nearrow g$. Then $g-g_{n} \searrow 0$ that gives $\lim _{n} \hat{s}\left(g_{n}\right)=\hat{s}(g)$ and $\hat{s}$ is $\sigma$-additive on $(G, u)$ as claimed.

Theorem 6.5. Let $E$ be an interval effect algebra with (BSP) and let $s$ be a state on $E$. Then there is a unique regular Borel probability measure, $\mu_{s}$, on $\mathcal{B}(\mathcal{S}(E))$ such that

$$
s(a)=\int_{\partial_{e} \mathcal{S}(E)} \hat{a}(x) \mathrm{d} \mu_{s}(x), \quad a \in E .
$$

Proof. Due to Theorem 6.3, we have a unique regular Borel probability measure $\mu_{s} \sim \delta_{s}$ such that (6.1) holds. The characterization of Bauer simplices, Alf, Thm II.4.1], says that then $\mu_{s}$ is a unique regular Borel probability measure $\mu_{s}$ on such that (6.1) holds and $\mu_{s}\left(\partial_{e} \mathcal{S}(E)\right)=1$. Hence, (6.2) holds.

We recall that Theorems 6.2 6.5 are applicable to every (i) effect algebra satisfying (RDP) and (BSP), respectively, (ii) MV-algebra and therefore, Theorem 6.5 generalizes the result of $\mathrm{Pan}, \mathrm{Kro}$. Since the state space of $\mathcal{E}(H)$ is not a simplex, the above theorems are not applicable for this important case. However, the next result shows that also in this case, every state $s$ on $\mathcal{E}(H)$ is an integral over some Bauer simplex, but the uniqueness of a regular Borel probability measure $\mu_{s}$ is not more guaranteed.

Theorem 6.6. Let $s$ be a state on an interval effect algebra $E$. Then there is a regular Borel probability measure, $\mu_{s}$, on the Borel $\sigma$-algebra $\mathcal{B}(\mathcal{S}(E))$ such that

$$
s(a)=\int_{\mathcal{S}(E)} \hat{a}(x) \mathrm{d} \mu_{s}(x), \quad a \in E .
$$


Proof. The set $\mathcal{S}(E)$ is non-void and a compact convex Hausdorff topological space. Given a state $s \in \mathcal{S}(E)$, by Theorem 6.2, $s$ can be uniquely extended to a state $\tilde{s}$ on $(\operatorname{Aff}(S(E)), 1)$. Since $(\operatorname{Aff}(\mathcal{S}(E)), 1))$ is a unital po-group that is a subgroup of the unital group $(\mathrm{C}(\mathcal{S}(E)), 1)$, but $(\mathrm{C}(\mathcal{S}(E)), 1)$ is an $\ell$-group. Therefore, this state $\tilde{s}$ can be by [Goo, Cor 4.3] extended to a state $s^{\prime}$ on the unital $\ell$-group $\mathrm{C}(\mathcal{S}(E), 1)$. But $(\mathrm{C}(\mathcal{S}(E)), 1)$ satisfies interpolation and $\Gamma(\mathrm{C}(\mathcal{S}(E)), 1)$ has (BSP), therefore by Theorem 6.5, there is a regular Borel probability measure $\nu_{s}$ on the Bauer simplex $\Omega=\mathcal{S}(\mathrm{C}(\mathcal{S}(E)), 1)$ such that

$$
f\left(s^{\prime}\right)=\int_{\partial_{e} \Omega} f(x) \mathrm{d} \nu_{s}(x), \quad f \in \Gamma(\mathrm{C}(\mathcal{S}(E)), 1) .
$$

Let $\epsilon: \mathcal{S}(E) \rightarrow \partial_{e} \mathcal{M}_{1}^{+}(\mathcal{S}(E))$ defined by (4.3). Then $\epsilon$ is a homeomorphism. For any $a \in E$, by [Goo, Cor 7.5], there is a unique continuous affine mapping $\tilde{a}: \mathcal{M}_{1}^{+}(\mathcal{S}(E)) \rightarrow[0,1]$ such that $\hat{a}=\tilde{a} \circ \epsilon$. Therefore, $\tilde{a} \in \Gamma(\mathrm{C}(\mathcal{S}(E)), 1)$ and $s(a)=\hat{a}(s)=\tilde{a}\left(\delta_{s}\right)$. The homeomorphism $\epsilon$ defines a regular Borel measure $\mu_{s}:=$ $\nu_{s} \circ \epsilon$ on $\mathcal{B}(\mathcal{S}(E))$, and using (6.4) and transformation of integrals, we have

$$
\begin{aligned}
s(a)=\tilde{a}(\epsilon(s)) & =\int_{\partial_{e} \Omega} \tilde{a}(y) \mathrm{d} \nu_{s}(y)=\int_{\epsilon(\mathcal{S}(E))} \hat{a} \circ \epsilon^{-1}(y) \mathrm{d} \nu_{s}(y) \\
& =\int_{\mathcal{S}(E)} \hat{a}(x) \mathrm{d} \mu_{s}(x) .
\end{aligned}
$$

This implies (6.3).

Remark 6.7. We note that reasons why we do not know to guarantee the uniqueness of $\mu_{s}$ in (6.3) are facts that $\mathcal{S}(E)$ is not necessarily a Bauer simplex nor a Choquet one, and $\operatorname{Aff}(\mathcal{S}(E))$ is sup-norm closed in $\mathrm{C}(\mathcal{S}(E))$ so we cannot extend a state on $(\operatorname{Aff}(\mathcal{S}(E)), 1)$ by continuity to a state on $(\mathrm{C}(\mathcal{S}(E)), 1)$.

Corollary 6.8. Let $E$ be an interval effect algebra with (BSP). The mapping $s \mapsto \mu_{s}$, where $\mu_{s}$ is a unique regular Borel probability measure satisfying (6.2), is an affine homeomorphism between $\mathcal{S}(E)$ and the set of regular Borel probability measures on $\mathcal{B}(S(E))$ endowed with the weak $k^{*}$ topology. A state $s$ on $E$ is extremal if and only if $\mu_{s}$ in (6.2) is extremal. In such a case, $\mu_{s}=\delta_{s}$.

Proof. The fact that that the mapping $s \mapsto \mu_{s}$ is affine and injective follows from Theorem [6.5] and (6.2). The surjectivity is clear because if $\mu$ is a regular Borel probability measure, then $\mu$ defines via (6.2) some state, $s$, on $E$. The continuity follows from [Alf, Thm II.4.1(iii)].

Since every Dirac measure $\delta_{s}$ is always a regular Borel probability measure, (6.2) entails that $s$ has to be extremal. Conversely, if $s$ is extremal, the uniqueness of $\mu_{s}$ yields that $\mu_{s}=\delta_{s}$.

It is worthy to recall that Corollary 6.8 shows that every (finitely additive) state on an interval effect algebra satisfying (BSP) is in a one-to-one correspondence with $(\sigma$-additive) regular Borel probability measure on some Borel $\sigma$-algebra trough formula (6.2). This observation is interesting because according to de Finetti, a probability measure is only a finitely additive measure, whilst by Kolmogorov [Kol] a probability measure is assumed to be $\sigma$-additive. (6.2) shows that there is a natural coexistence between both approaches. 
A famous result by Horn and Tarski [HoTa] states that every state on a Boolean subalgebra $A$ of a Boolean algebra $B$ can be extended to a state on $B$, of course not in unique way. E.g. the two-element Boolean subalgebra, $\{0,1\}$ has a unique state, 0 -1-valued, and it can be extended to many distinct states on $B$. This result was generalized for MV-algebras, see [Kro1, Thm 6]. In what follows, we generalize this result also for interval effect algebras, and this gives a variant of the Horn-Tarski Theorem.

Theorem 6.9. Let $E$ be an interval effect algebra and $F$ its sub-effect algebra. Then every state $s$ on $F$ can be extended to a state on $E$.

Proof. Let $E$ be an interval effect algebra and $F$ be its subalgebra. According to DvPu, Cor 1.4.5], $F$ is also an interval effect algebra. According to Remark 2.2 we can assume that $E=\Gamma(G, u)$, where $u$ is a universal strong unit for $E$. Then $F \cong \Gamma\left(H^{\prime}, u^{\prime}\right)$, where $H^{\prime}$ is a po-group with a universal strong unit $u^{\prime}$. Let $\iota_{F}$ be the embedding of $F$ into $E$ and let $\iota$ be an isomorphism of $\Gamma\left(H^{\prime}, u^{\prime}\right)$ onto $F$. Assume that $s$ is a state on $F$. Then $s^{\prime}=s \circ \iota$ is a state on $\Gamma\left(H^{\prime}, u^{\prime}\right)$. Applying again Remark 2.2. the state $s^{\prime}$ can be extended to a unique state, $s^{\prime \prime}$, on $\Gamma\left(H^{\prime}, u^{\prime}\right)$. On the other hand, the mapping $\iota$ can be extended to a unique group homomorphism, $\widehat{\iota}$, from $\left(H^{\prime}, u^{\prime}\right)$ into $(G, u)$ such that $\widehat{\iota}\left(u^{\prime}\right)=u$. Because $\iota$ was injective, so is $\widehat{\iota}$, and therefore, $\widehat{\iota}$ is an embedding. This entails that on the unital subgroup $\left(\widehat{\iota}\left(H^{\prime}\right), u\right)$ we have a state $\left.s^{\prime \prime \prime}=s^{\prime \prime} \circ \widehat{(i)}\right)^{-1}$ that is an extension of the original state $s$ on $F$. Applying [Goo, Cor 4.3], we see that $s^{\prime \prime \prime}$ can be extended to a state $\tilde{s}$ on $(G, u)$ so that, the restriction of $\tilde{s}$ onto $E=\Gamma(G, u)$ is an extension of a state $s$ on $F$.

Corollary 6.10. Let $E$ be an effect algebra satisfying (RDP) and let $F$ be its subalgebra. Then every state on $F$ can be extended to a state on $E$.

Proof. If $E$ satisfies (RDP), then $E$ is an interval effect algebra. The desired result now follows from Theorem 6.9.

\section{Conclusion}

Using techniques of simplices and affine continuous functions on a convex compact Hausdorff space, we have showed that every state on an interval effect algebra, i.e. on an interval $[0, u]$ of a unital po-group $(G, u)$, can be represented by an integral, Theorems 6.3 6.6. A sufficient condition for this is the condition that the state space, $\mathcal{S}(E)$, of an interval effect algebra $E$ is a Choquet simplex, Theorem 6.4. In this case it is even a Bauer simplex, Theorem 6.5 and there is a one-to-one correspondence between the set of all states on $E$ and the set of all regular Borel probability measures on the Borel $\sigma$-algebra $\mathcal{B}(\mathcal{S}(E)$ ), Corollary 6.8

On the other side, an important example for mathematical foundations of quantum mechanics, the state space of the effect algebra $\mathcal{E}(H)$, the set of all Hermitian operators on a Hilbert space $H$ that are between the zero operator and the identity, is not a simplex, but also in this case we can represent every state as an integral through a regular Borel probability measure over some Bauer simplex, Theorem 6.6. however in this case, the uniqueness of a Borel probability measure is not more guaranteed.

In addition, we have proved a variant of the Horn-Tarski Theorem that every state on a subalgebra of an unital effect algebra can be extended to a state on the interval effect algebra, Theorem 6.9. 


\section{REFERENCES}

[Alf] E.M. Alfsen, "Compact Convex Sets and Boundary Integrals", Springer-Verlag, Berlin, 1971.

[AlSc] E.M. Alfsen, F.W. Schultz, "State Spaces of Operator Algebras", Birhauser, 2001.

[Cha] C.C. Chang, Algebraic analysis of many valued logics, Trans. Amer. Math. Soc. 88 (1958), 467-490.

[CDM] R. Cignoli, I.M.L. D'Ottaviano, D. Mundici, "Algebraic Foundations of Many-valued Reasoning", Kluwer Academic Publ., Dordrecht, 2000.

[DuSc] N. Dunford, J.T. Schwartz, "Linear Operators, Part I," Interscience, New York-London, 1958.

[Dvu] A. Dvurečenskij, “Gleason's Theorem and Its Applications”, Kluwer Academic Publisher, Dordrecht/Boston/London, 1993,

[DvVe1] A. Dvurečenskij, T. Vetterlein, Pseudoeffect algebras. I. Basic properties, Inter. J. Theor. Phys. 40 (2001), 685-701.

[DvVe2] A. Dvurečenskij, T. Vetterlein, Pseudoeffect algebras. II. Group representation, Inter. J. Theor. Phys. 40 (2001), 703-726.

[DvPu] A. Dvurečenskij, S. Pulmannová, "New Trends in Quantum Structures", Kluwer Acad. Publ., Dordrecht, Ister Science, Bratislava, 2000.

[FoBe] D.J. Foulis, M.K. Bennett, Effect algebras and unsharp quantum logics, Found. Phys. 24 (1994), 1325-1346.

[Gle] A.M. Gleason, Measures on the closed subspaces of a Hilbert space, J. Math. Mech. 6 (1957), 885-893.

[Goo] K.R. Goodearl, "Partially Ordered Abelian Groups with Interpolation", Math. Surveys and Monographs No. 20, Amer. Math. Soc., Providence, Rhode Island, 1986.

[Hal] P.R. Halmos, "Measure Theory", Springer-Verlag, New York, Heidelberg, Berlin, 1988.

[HoTa] A. Horn, A. Tarski, Measures on Boolean algebras, Trans. Amer. Math. Soc. 64 (1948), 467-497.

[Kol] A.N. Kolmogorov, "Grundbegriffe der Wahrscheinlichkeitsrechnung", Berlin, 1933.

[KoCh] F. Kôpka, F. Chovanec, D-posets, Math. Slovaca 44 (1994), 21-34.

[Kro] T. Kroupa, Every state on semisimple $M V$-algebra is integral. Fuzzy Sets and Systems 157 (2006), 2771-2782.

[Kro1] T. Kroupa, Representation and extension of states on $M V$-algebras. Archive Math. Logic 45 (2006), 381-392.

[LuZa] W.A.J. Luxemburg, A.C. Zaanen, "Riesz Spaces, I" North-Holland, Amsterdam, London, 1971.

[Mun] D. Mundici, Interpretation of $A F C^{*}$-algebras in Eukasiewicz sentential calculus, J. Funct. Anal. 65 (1986), 15-63.

[Pan] G. Panti, Invariant measures in free MV-algebras, Comm. Algebra 36 (2008), 2849-2861.

[Phe] R.R. Phelps, "Lectures on Choquet's Theorem", Van Nostrand, Princeton, 1965.

[Rav] K. Ravindran, On a structure theory of effect algebras, PhD thesis, Kansas State Univ., Manhattan, Kansas, 1996. 